\title{
PERFORMANCE EVALUATION OF VARIOUS COOPERATIVE SPECTRUM SENSING ALGORITHMS FOR REDUCING COMMUNICATION OVERHEADS
}

\author{
Renu Kumari ${ }^{1}$ \\ ${ }^{I}$ Department of Electronics and Communication, NIT Patna
}

\begin{abstract}
Due to the unavoidable use of mobile phone and another data communication as a result we face the problem of spectrum scarcity. Within the current spectrum regulatory framework, however, all of the frequency bands are exclusively allocated to specific services and no violation from unlicensed users is allowed.But as we know that most of the time band is either not utilized or underutilized. The problem of spectrum scarcity and spectrum under-utilization can be solved by the use of cognitive radio spectrum sensing. But due to deep fading and shadowing spectrum sensing highly degrades. As all we know that if secondary users cooperate with each other, spectrum efficiency and most of the other problem such as hidden terminal problem, loss in spectrum utilization can be solved. A comparison study is made in between various algorithms which is proposed to overcome these problems in this paper.
\end{abstract}

Keywords-Cognitive radio, spectrum sensing, , cooperative sensing,multi-path fading, communication overheads. ****

\section{INTRODUCTION}

With the increased in wireless technologies in recent year increases the demand for wireless services with high quality and data rate. As we know that the radio frequency spectrum is a limited resource with great importance hence problem of spectrum scarcity increases. According toFederal Communications Commission (FCC)[1] have shown that $70 \%$ of the allocated spectrum in US is not utilized. Consequently, innovative techniques that more efficiently utilize the available spectrum are needed. To mitigate this problem cognitive radio was first proposed by Mitola[2], for better utilization of spectrum bands by both primary and secondary users. According to Mitola Cognitive radio is defined as" The point in which wireless personal digital assistants (PDAs) and the related networks are sufficiently computationally intelligent about radio resources and related computer-to-computer communications to: (a) detect user communications needs as a function of use context, and (b) to provide radio resources and wireless services most appropriate to those needs."

In cognitive radio network each secondary user senses the presence or absence of the primary user in order to avoid of interference before utilization. For better sensing result each and every secondary form a group and so such groups sends their result to a common fusion Centre where there results are combined to make a final decision whether the primary user is present or not. With the use of cooperative spectrum sensing effect of deep fading and shadowing is overcome but with the increase of additional communicational overhead. It has been shown that the spectrum sensing performance can be greatly improved with an increase of the number of cooperative users[3]-[5]. In paper [6] a complete survey of cooperative spectrum sensing and various factors that can affect the sensing is discussed thoroughly. There are various methods are proposed to overcome the communication overhead. To decrease secondary users' transmission time and consuming their limited energy a novel adaptive cooperative spectrum sensing algorithm [7] is proposed by Jingqun Song. This algorithm adjusts its sensing time according to channel usage characteristics and the number of secondary users in cooperative spectrum sensing.In this paper [8], author Chia-han Lee, proposed two energy efficient technique to overcome communication overheads. In first energy efficient scheme named confidence voting algorithm in which user can cast their vote if it has confidence hence saved transmission energy and time and Second energy efficient technique is ClusterCollect-Forward (CCF) which takes the advantage of cooperation of collect and forward over broadcasting hence $65 \%$ to $95 \%$ transmission energy can be saved if cluster is chosen carefully.

The rest of this paper is organized as follows. Section II explains the various algorithms which increases performances by reducing the overall communication overhead. The results are investigated, analyzed and compared with existing methods in Section III.Finally, this paper is concluded by providing some final remarks in Section IV.

\section{SOME PROPOSED ALGORITHMS REDUCE COMMUNICATION OVERHEAD} TO

\subsection{Weight Combining Algorithm}

In weight combining method [9], each and every secondary user is given weight by the fusion Center according to 
received energy of the primary signal and the path loss which occur due to the distance between primary and secondary user. Actually, Fusion Centre(FC) know the distance between each secondary user and primary user, so it gives less weight if the Path-loss is more and higher weight if the received energy by the secondary user is strong. After combining these weights FC can be identified that which secondary user gives the adequate result hence those user whose gets less weight need not to send their result to the Fusion Centre in future hence the communication overheads can be reduced up to a large extends.

\subsection{Communication Overhead Aware Method}

In this algorithm [10] a smoothing factor is used which play a crucial role in between information exchange between Fusion Center and Secondary Users(SU). According to this, when SUs sense then the result is compare with the previous result and if the difference is small then no result will be sent to the Fusion Centre and the SU keeps silent in this sensing interval otherwise the result will be sent to FC .Now if FC will not receive any decision from SU in that case it will make decision on the basis of the previous result for that secondary user otherwise when it receive local sensing from secondary user then it upgrade local sensing threshold and uses it to make final decision to that secondary user. Hence we see that the sensing time and unnecessary spectrum utilization reduce up to a large extend.

\subsection{Bargaining Based Pairwise Cooperative Spectrum Sensing (BBPCSS)}

To increase the sensing efficiency BBPCSS [11] is proposed by Miaho. In this algorithm, in order the mitigate the problem of multipath-fading and shadowing effect cognitive radio are grouped into pair for spectrum sensing instead of individual sensing. In each pair, each cognitive radio $\mathrm{CR}$ users can bargain with each other over the sensing time division, and thus save the sensing time for data transmission.

\subsection{Improved Censor Based Cooperative Spectrum}

\section{Sensing}

This scheme is used to reduce the loss of spectrum utilization. This algorithm [12] uses double threshold method to find the presence and absence of primary user. Those users whose collected energy is above and below the two thresholds send their one -bit decision to the FCIn this case we see that if collected energy is above upper threshold then primary user is not present or if collected energy is below the low threshold then primary user is present otherwise no decision about primary user will be made if values lie in between upper and lower threshold and SUs send nothing to FC, butin improved censor-based scheme if the collected energy lies between these two thresholds SU sends it to Fusion center with a probability of $\mathrm{p}$ and nothing send with a probability of 1-p.

\subsection{Cooperative Wideband Spectrumsensing}

\section{Framework with LRT-Based Optimal Fusion Rule}

With the help of this algorithm [13], problem like Challenges like low signal-to-noise ratio (SNR), fading and inaccuracy in noise power estimation can be solved up to an extent. First of all, each secondary user calculate the power of each channel and using BEED (Bayesian estimationbased energy detection ) method to determine the occupancy of each channel and the decision is sent to the Fusion Center. The final decision is made by combining the local decisions by fusion Center and for optimal rule LRT (Likelihood Ratio Test).

\section{COMPARISON BETWEEN DIFFERENT ALGORITHMS}

\begin{tabular}{|c|c|c|c|c|}
\hline ALGORITHMS & $\begin{array}{l}\text { NOISE } \\
\text { UNCERTAINTY }\end{array}$ & $\begin{array}{l}\text { SPECTRUM } \\
\text { UTILIZATION }\end{array}$ & $\begin{array}{l}\text { SENSING } \\
\text { TIMING }\end{array}$ & $\begin{array}{l}\text { PERFORMANCE } \\
\text { IMPROVEMENT }\end{array}$ \\
\hline Weight combining & $\begin{array}{l}\text { GREATER } \\
\text { ROBUSTNESS }\end{array}$ & increased & REDUCED & $68.25 \%$ OF EGC \\
\hline $\begin{array}{l}\text { Improved censor } \\
\text { based }\end{array}$ & Suffer & $\begin{array}{l}\text { Degrade the loss } \\
\text { of spectrum } \\
\text { utilization }\end{array}$ & REDUCED & $\begin{array}{l}8 \mathrm{TO} 17 \% \text { increase } \\
\text { in agility gain }\end{array}$ \\
\hline $\begin{array}{l}\text { COOPERATIVE } \\
\text { WIDEBAND } \\
\text { SPECTRUM } \\
\text { SENSING }\end{array}$ & $\begin{array}{l}\text { GREATER } \\
\text { ROBUSTNESS }\end{array}$ & No change & reduced & $\begin{array}{l}\text { Handle the } \\
\text { problem of low } \\
\text { SNR, fading and } \\
\text { inaccuracy in noise } \\
\text { power estimation }\end{array}$ \\
\hline $\begin{array}{l}\text { COMMUNICATION } \\
\text { OVERHEAD }\end{array}$ & reduced & increased & increased & $\begin{array}{l}20 \% \\
\text { communication } \\
\text { overhead reduced }\end{array}$ \\
\hline BBPCSS & $\begin{array}{l}\text { Reduced } \\
\text { increasing } \\
\text { spectrum } \\
\text { accuracy }\end{array}$ & improved & reduced & Utility improved \\
\hline
\end{tabular}




\section{CONCLUSIONS}

Spectrum sensing is the emerging key technology for the various applications of $\mathrm{CR}$ networks in commercial, public safety, and particularly military areas. Interference avoidance, fading, communication overheads are challenging problems for spectrum sensing which reduces sensing efficiency. In this paper, we presented a reasonable cooperative sensing scheme using various ways to give a joint consideration of these issues.

\section{REFERENCES}

[1]. Federal Communications Commission, "Notice of proposed rulemaking: Unlicensed operation in the TV broadcast bands," ET Docket No. 04-186 (FCC 04-113), May 2004.

[2]. I. Mitola, J. and J. Maguire, G. Q., "Cognitive radio: making software radios more personal," IEEE Personal Commun. Mag., vol. 6, no. 4, pp. 13-18, Aug. 1999.

[3]. C. Sun, W. Zhang, and K. B. Letaief, "Cluster-based cooperative spectrum sensing for cognitive radio systems," in Proc. IEEE Int. Conf. Commun., Glasgow, Scotland, UK, June 24-28, 2007, pp. 2511-2515.

[4]. G. Ganesan and Y. G. Li, "Cooperative spectrum sensing in cognitive radio networks," in Proc. IEEE Symp. New Frontiers in Dynamic Spectrum Access Networks (DySPAN'05), Baltimore, USA, Nov. 8-11, 2005, pp. 137143.

[5]. S. M. Mishra, A. Sahai, and R. Brodersen, "Cooperative sensing among cognitive radios," in Proc. IEEE Int. Conf. Commun., Turkey, June 2006,

[6]. Ian F. Akyildiz, Brandon F. Lo and RavikumarBalakrishnan," Cooperative spectrum sensing in cognitive radio networks: A survey", ELSEVIER,physical communication 4, pp.40-62,2011.

[7]. Jingqun Song1, Huying Cai2, ZhiyongFeng" A Novel Cooperative Spectrum Sensing Scheme Based on Channelusage in Cognitive Radio Networks" This work is sponsored by the National Basic Research Program of China (973 Program) (No.2009CB320406), the National High Technology Research and Development Program of China (863 Program) (No. 2009AA011802),IEEE,PP.1-4,2009

[8]. Chia-han Lee and Wayne Wolf," Energy Efficient Techniques for Cooperative Spectrum Sensing in Cognitive Radios', IEEECommunications Society, pp.-968-972, 2008.

[9]. Mohammad Iqbal Bin Shahid and JoarderKamruzzaman," Weighted Soft Decision for Cooperative Sensing in Cognitive Radio Networks', IEEE, ICON, pp.1-6,2008,

[10]. Nannan Zhang, Huifang Chen and Lei Xie," A Communication-overhead-aware Cooperative Spectrum Sensing Scheme in Cognitive Radio Networks", This work was partly supported by National Natural Science Foundationof China (No. 61071127), Science and Technology Department of Zhejiang Province (No. 2011R10035),IEEE,pp.1-5 ,2012.

[11]. Miao Pan, Yuguang Fang," Bargaining Based Pairwise Cooperative Spectrum Sensing for Cognitive Radio Networks" National Science Foundation under grants CNS,IEEE, pp. 1-7,2008.
[12]. Chen Guo, Wenbo Wang, Tao Peng," Agility Improvements by Censor-Based Cooperative Spectrum Sensing in Cognitive Radio Networks", pp. 1-5

[13]. VahidJamali, Ramezan Ali SadeghZadeh, S. Hamid Safavi and SoheilSalari,', Optimal Cooperative Wideband Spectrum Sensingin Cognitive Radio Networks", IEEE, ICUFN, pp. 371-374, 2011. 